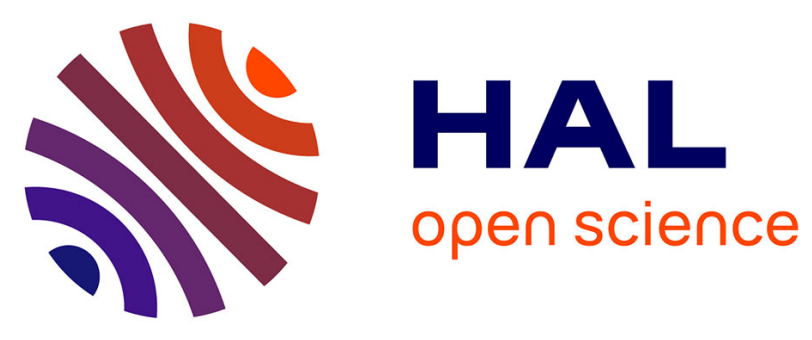

\title{
Nonlinear Spectroscopy with X-Ray Two-Photon Absorption in Metallic Copper
}

Kenji Tamasaku, Eiji Shigemasa, Yuichi Inubushi, Ichiro Inoue, Taito Osaka, Tetsuo Katayama, Makina Yabashi, Akihiro Koide, Toshihiko Yokoyama, Tetsuya Ishikawa

\section{To cite this version:}

Kenji Tamasaku, Eiji Shigemasa, Yuichi Inubushi, Ichiro Inoue, Taito Osaka, et al.. Nonlinear Spectroscopy with X-Ray Two-Photon Absorption in Metallic Copper. Physical Review Letters, 2018, 121 (8), pp.083901. 10.1103/PhysRevLett.121.083901 . hal-01879726

\section{HAL Id: hal-01879726}

https://hal-univ-rennes1.archives-ouvertes.fr/hal-01879726

Submitted on 23 Jan 2019

HAL is a multi-disciplinary open access archive for the deposit and dissemination of scientific research documents, whether they are published or not. The documents may come from teaching and research institutions in France or abroad, or from public or private research centers.
L'archive ouverte pluridisciplinaire HAL, est destinée au dépôt et à la diffusion de documents scientifiques de niveau recherche, publiés ou non, émanant des établissements d'enseignement et de recherche français ou étrangers, des laboratoires publics ou privés. 


\section{Nonlinear spectroscopy with x-ray two-photon absorption in metallic copper}

Kenji Tamasaku, ${ }^{1, *}$ Eiji Shigemasa, ${ }^{2}$ Yuichi Inubushi, ${ }^{3}$ Ichiro Inoue, ${ }^{1}$ Taito Osaka, ${ }^{1}$ Tetsuo Katayama, ${ }^{3}$ Makina Yabashi, ${ }^{1,3}$ Akihiro Koide, ${ }^{2,4}$ Toshihiko Yokoyama, ${ }^{2}$ and Tetsuya Ishikawa ${ }^{1}$

${ }^{1}$ RIKEN SPring-8 Center, 1-1-1 Kouto, Sayo-cho, Sayo-gun, Hyogo 679-5148, Japan.

${ }^{2}$ Institute for Molecular Science, 38 Nishigo-Naka, Myodaiji, Okazaki-shi, Nagoya 444-8585, Japan. ${ }^{3}$ Japan Synchrotron Radiation Research Institute,

1-1-1 Kouto, Sayo-cho, Sayo-gun, Hyogo 679-5198, Japan.

${ }^{4}$ Univ Rennes, CNRS, IPR (Institut de Physique de Rennes) - UMR 6251, F-35000 Rennes, France (Dated: August 24, 2018)

X-ray two-photon absorption (TPA) spectrum of metallic copper is measured using a free-electron laser (XFEL). The spectrum differs from that measured by the conventional one-photon absorption (OPA), and characterized by a peak below the Fermi level, which is assigned to the transition to the $3 \mathrm{~d}$ state. The impact of the XFEL pulse on the OPA spectrum is discussed by analyzing the pulse-energy dependence, which indicates that the intrinsic TPA spectrum is measured. 


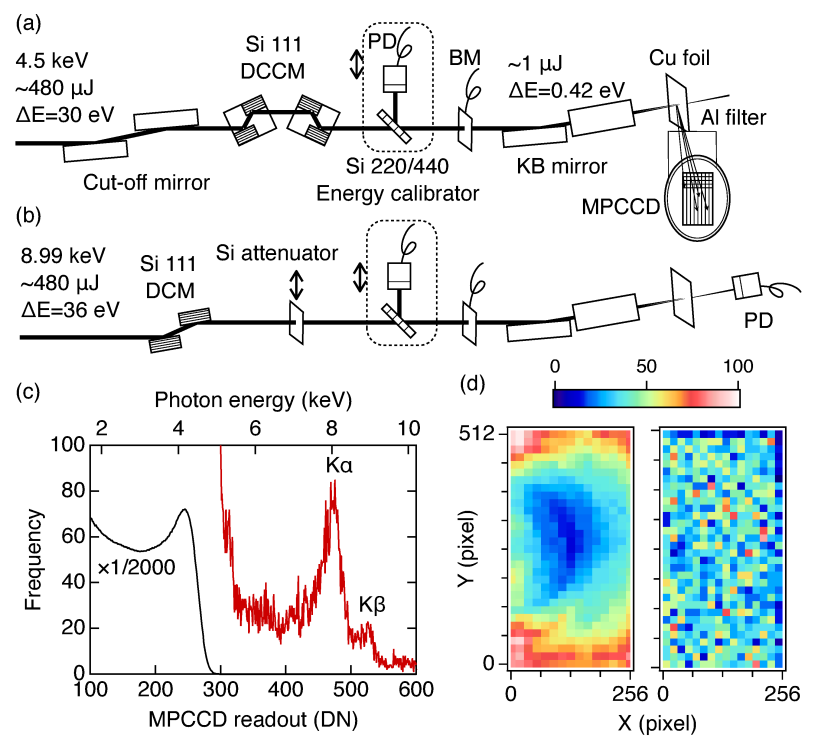

FIG. 1. (a,b) Schematic experimental setup for the TPA (a) and the OPA (b) spectroscopy. See text for details. (c) The histogram of the MPCCD readout. (d) The event distribution on the MPCCD chip around $4.5 \mathrm{keV}$ (left panel) and $8 \mathrm{keV}$ (right panel). Color bar represents the normalized frequency. Each bin shows the average over 16 by 16 pixels.

X-ray absorption spectroscopy (XAS) is a powerful tool for element selective study on local structure and electronic states in physical, chemical and biological disciplines [1]. In addition to OPA used in XAS, the advent of XFEL [2, 3] makes it possible to observe nonlinear TPA process [4-7]. The TPA transition can access different parity states from those probed with OPA $[5,8]$, and is expected to uncover hidden information to the conventional XAS. However, the low-efficiency TPA process has been observed using intense pink beam at the cost of severe sample damage. It is still challenging to perform x-ray TPA spectroscopy using monochromatic beam, and to apply it to a study of the intrinsic electronic structure without excess heating and core-hole creation.

The damage process is triggered predominantly by the OPA process $[4,5,9-13]$. The OPA probability of an atom can be written as $R=\sigma_{\text {tot }} F$, where $\sigma_{\text {tot }}$ is the total OPA cross-section, and $F$ is the fluence (photon number per unit area) [13]. When $F$ exceeds the critical fluence, $F_{\mathrm{c}}=1 / \sigma_{\mathrm{tot}}$, the atom will be sequentially photo-ionized during the whole pulse duration. As a result, suppression of the OPA $[9,14,15]$ and the TPA [5] processes are observed around $R=1$. Thus, $R \ll 1$ should be satisfied for spectroscopic applications, otherwise the spectrum could be highly contaminated with populated highly charged atoms.

In this Letter, we report x-ray TPA spectrum of metallic copper. In the present study, we satisfied $R \ll 1$ using lower pulse energies and looser focusing than the previous report [5], compensated the peak intensity reduction by short pulse, and employed an efficient detecting system with larger solid-angle coverage for the weaker TPA signal. We also report an experimental study on the threshold for x-ray absorption spectroscopy below which the intrinsic absorption spectrum can be measured, showing that an absorbed energy density is a better measure of the threshold for solids than $F_{\mathrm{c}}$.

The fluorescence-yield TPA spectrum was measured at SACLA BL3 using a 4.5-keV beam with a full width at halfmaximum (FWHM) pulse duration of 8 fs [16], a bandwidth of $30 \mathrm{eV}$ (FWHM), and an average pulse energy of $480 \mu \mathrm{J}$ [Fig. 1(a)]. The higher-harmonic radiation was suppressed by a double-bounce mirror with a cut-off energy of 7.5 keV. Then, a double channel-cut monochromator (DCCM) with the Si 111 reflection selected single photon energy with a 0.42-eV bandwidth (FWHM). The second harmonic radiation was rejected by the forbidden 222 reflection at DCCM (see Supplemental Material [17]). Residual harmonics content in the beam can also contribute with an OPA signal to the fluorescence signal. However, the OPA signal should have a linear dependence and can be easily disentangled from the TPA signal, which has a quadratic dependence that was possible to derive in the present experiment as is shown later.

The Kirkpatrick-Baez (KB) mirror focused the pump beam on a 10- $\mu$ m-thick copper foil. The focus size was measured to be $1.5(\mathrm{H}) \times 1.6(\mathrm{~V}) \mu \mathrm{m}^{2}(\mathrm{FWHM})$ by knife-edge scans [18]. The pulse energy was monitored in front of the KB mirror by a calibrated beam monitor (BM) [19]. The copper foil was shifted continuously to expose fresh surface. To measure the fluorescence at right angle to the pump beam, the foil was tilted by 30 degrees from the normal incidence This geometry suppresses the background due to elastic and the Compton scattering. A multi-port 


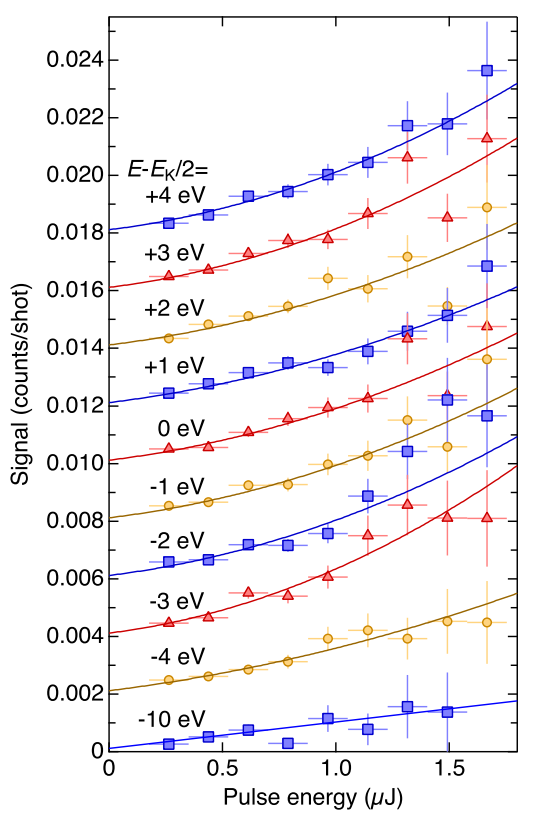

FIG. 2. Pulse-energy dependence of TPA fluorescence of metallic copper at several pump photon energies. Vertical and horizontal bars indicate the statistical error and the range of the pulse energy for each bin, respectively. Solid line is a fit by a quadratic function with a linear and a constant terms. The pump photon energy is labeled deviation from $E_{\mathrm{K}} / 2$.

charge-coupled device (MPCCD Phase-IIIL) [20] detected the shot-by-shot fluorescence signal emitted into a solid angle of as large as $0.034 \mathrm{sr}$.

The OPA transmission spectra around the $\mathrm{K}$ edge $\left(E_{\mathrm{K}}=8.98 \mathrm{keV}\right)$ were also measured to calibrate the photon energy of the TPA spectrum, and to estimate the threshold to measure the intrinsic absorption spectrum. The experimental setup was the same as the TPA spectroscopy, except that a Si(111) double-crystal monochromator (DCM) was used instead of DCCM and the cut-off mirror [Fig. 1(b)]. The transmission was measured by a photodiode (PD). A 0.2-mm-thick silicon attenuator was inserted for the spectra under lower pulse energies.

The procedure to calibrate the abscissa axis of the TPA spectrum to the OPA spectrum is as follows. After measuring the OPA spectrum, DCM was set to $E_{\mathrm{K}}$. A thin silicon crystal was inserted downstream, and was adjusted to produce the 440 reflection in the transmission geometry. Then, DCCM and the cut-off mirror replaced DCM, and DCCM was tuned to make the crystal produce the 220 reflection at the same Bragg angle, where the pump photon energy is exactly $E_{\mathrm{K}} / 2$.

Figure 1(c) shows the histogram of the MPCCD readout for $8.2 \times 10^{6}$ shots. The count rate is very low, and the readout value can be regarded as each photon energy. The conversion coefficient is determined by the K $\alpha$ fluorescence measured during the OPA experiment. The strong peak around $4 \mathrm{keV}$ corresponds to the elastically scattered pump $\mathrm{x}$ rays. Charge sharing over pixels shifts the peak position from $4.5 \mathrm{keV}$, and broadens the peak. In the higher photon energy region (the readout $>320$ ), a simple droplet algorithm [21] was applied to correct the charge sharing effect, which recovered clear fluorescence lines, $\mathrm{K} \alpha$ and $\mathrm{K} \beta$.

In the TPA measurement, it is important to eliminate pile up, where two pump photons accidentally hit the same pixel. The pile up is a "two-photon" process, and could produce a fake signal near the fluorescence lines. The pile-up probability is quadratic to the $4.5-\mathrm{keV}$ photon number, so it can be efficiently suppressed by inserting an aluminum filter in front of MPCCD. The attenuation was adjusted by checking the signal distribution on the MPCCD [Fig. 1(d)]. The $4.5-\mathrm{keV}$ events are not observed in the central region, where the scatting angle becomes 90 degrees. On the other hand, the $8-\mathrm{keV}$ signals scatter uniformly, which indicates that they are not of the pile-up origin.

Figure 2 shows the pump photon-energy and the pulse-energy dependence of the signal rate with the MPCCD readout between 380 and 550. Since the pump photon energy is far from $E_{\mathrm{K}}$, the self-absorption effect, which must be corrected for the fluorescence-yield OPA spectrum, can be ignored. The average pulse energy was only $1 \mu \mathrm{J}$ after DCCM. Alternatively, the 8-fs short pulse ensured enough peak intensities around $5 \times 10^{15} \mathrm{~W} / \mathrm{cm}^{2}$ to yield the measurable TPA signal. The peak intensity is still two to four orders magnitude lower than the previous reports [4-7].

The super-linear dependence in Fig. 2 is reproduced well by a quadratic function. The quadratic term represents 

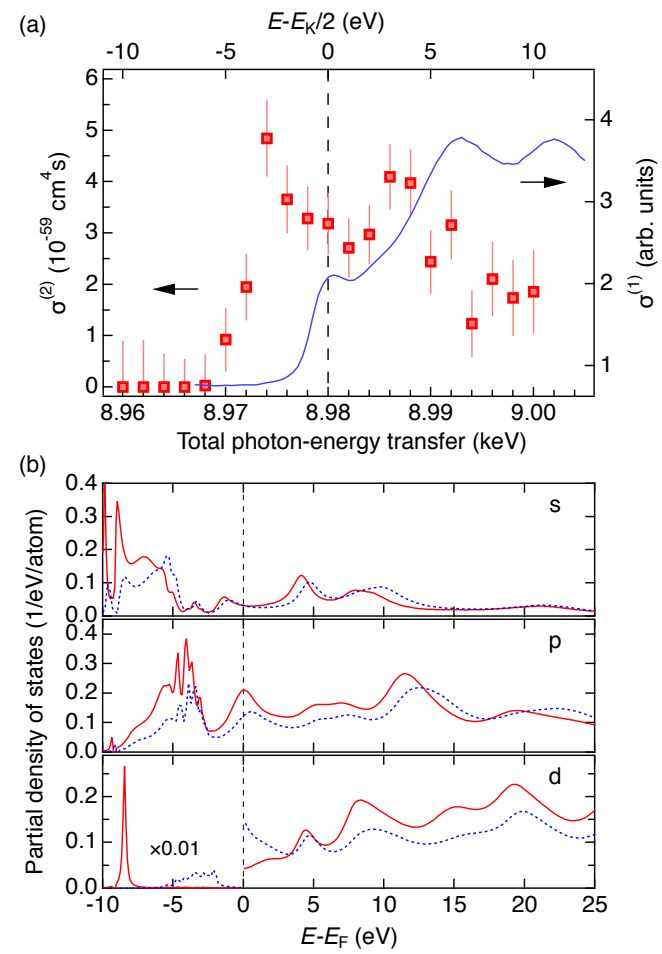

FIG. 3. (a) TPA (squares) and OPA (solid line) spectra of metallic copper. Vertical bars represent the fitting error. Vertical dashed line indicates the Fermi level threshold $\left(E_{\mathrm{K}}\right)$. (b) Partial density of states on the atom with a K-shell core hole (solid line) and the atom in the ground state. The symmetry of state is labeled on each panel.

the fluorescence by TPA. The quadratic contribution suddenly diminishes below $E-E_{\mathrm{K}} / 2=-4 \mathrm{eV}$. The linear contribution, which is common to all curves, is due to the OPA process by the residual third harmonic radiation and the undiscriminated pump photons. The latter is due to the insufficient photon-energy resolution of MPCCD to discriminate completely the $4.5-\mathrm{keV}$ pump from the $8-\mathrm{keV}$ fluorescence. There remains in addition a small constant background. Measurement under a dark condition confirms that cosmic rays produce $1.16 \times 10^{-4}$ events/frame within the signal region, and account for the background.

Figure 3(a) shows the fluorescence-yield TPA spectrum of metallic copper, i.e., the pump photon-energy dependence of the TPA cross-section, $\sigma^{(2)}$, which is deduced from the quadratic coefficient of the pulse-energy dependence $[5,6]$. The magnitude of $\sigma^{(2)}$ is found to be comparable to prediction by a simple $Z^{-6}$ scaling for hydrogen-like atoms [22]. Here, $Z$ is the atomic number. The TPA cross-section of hydrogen-like copper at $E_{\mathrm{K}}$ is calculated to be $\sigma^{(2)}(Z=29)=2.1 \times 10^{-59} \mathrm{~cm}^{4} \mathrm{~s}$ using $\sigma^{(2)}(Z=1)=1.27 \times 10^{-50} \mathrm{~cm}^{4} \mathrm{~s}$ [23]. At present, the knowledge of TPA spectra is very limited. The theoretical calculations are available only for gas phase [24, 25]. The previous experiments implied that the TPA process has a threshold $[4,6]$. The present study reveals the whole TPA structure: a sharp edge, corresponding to the change in the pulse-energy dependence at $E-E_{\mathrm{K}} / 2=-4 \mathrm{eV}$ (Fig. 2), followed by a peak. Surprisingly, the peak lies below $E_{\mathrm{K}}$, i.e., the Fermi level threshold.

Excitation to the states below the Fermi energy is prohibited strictly by the Pauli principle. However, when the promoted $1 \mathrm{~s}$ electron is assumed to enter the $3 \mathrm{~d}$ band, which is dipole-forbidden for OPA but allowed for TPA, the peak below the Fermi level threshold can be explained by the core-hole potential. The 3d electron feels stronger potential than $4 \mathrm{p}$, because the $3 \mathrm{~d}$ orbital is closer to the nucleus. The $3 \mathrm{~d}$ orbital is stabilized by the less-screened nucleus in the presence of the K-shell hole, which reduces the total energy needed to photo-excite the 1s electron to the $3 \mathrm{~d}$ band. In addition, the energy shift of $6 \mathrm{eV}$ between the peak and the Fermi level (Fig. 2) agrees with the theoretical shift of $6.3 \mathrm{eV}$ for the occupied $3 \mathrm{~d}$ band calculated by a multiple scattering code, FEFF [26], and $6.9 \mathrm{eV}$ by a band calculation code, VASP [27], which support the above picture.

In fact, a weak peak with about $1 \%$ intensity is often observed in the pre-edge region of the OPA spectrum of $3 \mathrm{~d}$ transition metal compounds $[1,28]$. The pre-edge peak consists of two contributions: the quadrupole transition from $1 \mathrm{~s}$ to the $3 \mathrm{~d}$ band and the dipole transition from $1 \mathrm{~s}$ to the $4 \mathrm{p}$ states hybridized into the $3 \mathrm{~d}$ band. However, no pre-edge peak is visible in Fig. 3(a). The absence of pre-edge peak is considered due to the low density of states for the empty $3 \mathrm{~d}$ band in metallic copper [Fig. 3(b)]. The prominent peak indicates higher sensitivity of x-ray TPA spectroscopy 
to the empty 3d band than the conventional XAS using OPA. The spectral weight of the peak is considered to be proportional to the density of states for the empty $3 \mathrm{~d}$ band, and can be used for quantitative analysis. In contrast to the pre-edge peak in the OPA spectrum, there is no complexity to distinguish the direct quadrupole contribution from the dipole transition to the hybridized $4 \mathrm{p}$ states.

The TPA spectrum shape can be explained by a theory proposed for metals with incomplete shell, such as the 4f system [28, 29]. In this model, the sharp Fermi level threshold disappears and sharp peaks appear below the threshold. Moreover, the peak shows power-law divergence due to the orthogonality catastrophe [30], which has successfully explained the similar divergence at the Fermi level threshold observed in the absorption and the innershell photo-emission spectra of simple metals. The TPA spectrum reveals for the first time the unique spectral feature in the $3 \mathrm{~d}$ transition metals.

In the latter part, we discuss the effect of the pump pulse on the TPA spectrum. The critical fluence is calculated to be $F_{\mathrm{c}}=3.8 \times 10^{19}$ photons $/ \mathrm{cm}^{2}$ at $E_{\mathrm{K}} / 2=4.5 \mathrm{keV}$ [31], and the OPA probability during the TPA experiment to be only $R=0.15 \%$. Nevertheless, faint molten imprints (damage) were observed on the sample surface. These are considered due to an impact process [32], where the fast electron created by photo-ionization further ionizes surrounding atoms. Numerical simulation for diamonds shows that a single photo-electron excites a number of secondary electrons within $10 \mathrm{fs}$ [33], which is comparable to the present pulse duration of 8 fs. The impact process may affect the absorption spectra of solids as a result of, for example, temperature elevation and new transition to empty states created in the valence band.

At present, no theoretical study is available to predict the effect of impact process on the absorption spectrum or the threshold to measure the intrinsic absorption spectrum. However, they can be evaluated experimentally from the pulse-energy dependence of the OPA transmission spectra shown in Fig. 4(a). The spectrum starts to deviate around the first peak. As the pulse energy increases, the deviated region spreads toward the higher photon-energy side, and the edge shifts. On the othrer hand, no change is observed in the pre-edge region up to $10 \mu \mathrm{J}$.

In the present study, the threshold is estimated from the onset of the spectral change. The raw transmission spectrum reflects the averaged absorption coefficient, $\bar{\mu}$, since the beam intensity, $I$, attenuates as it propagates in the sample. The intensity attenuation may be written as:

$$
\frac{d I}{d z}=-\mu(u) I
$$

where $z$ is the distance from the surface along the beam axis. Here, an absorbed energy density, $u=I \mu$, is introduced, because the number of the secondary electron depends on $u$ rather than $F$. Note that the temporal evolution of $\mu$ is ignored for simplicity. The transmittance, $T$, is calculated by integrating Eq. (1).

The inverse problem to determine $\mu(u)$ from $T$ was solved numerically by fitting of calculated $T$ using Eq. (1) to the measured pulse-energy dependence. The fitting parameters were the attenuation coefficient, $\mu$, defined at twenty $u$ points with equal intervals on the logarithmic scale. Between the points, $\mu$ was interpolated linearly. The fitting converged well under a reasonable assumption that $\mu$ is a monotonic function of $u$. Each transmittance data includes redundant information about $\mu$ over a range of $u$, which serves for stable fitting.

Figure 4(b) shows $\mu(u)$ estimated at the first peak A $(8.9805 \mathrm{keV})$ and the second peak B $(8.9925 \mathrm{keV})$ in the OPA spectrum together with $\bar{\mu}=-\ln (T) / t$, where $t$ is the sample thickness along the $z$ direction. From the $u$ dependence at $\mathrm{A}$, the threshold is determined to be $u_{\text {thes }}=0.02 \mu \mathrm{J} / \mu \mathrm{m}^{3}(1.5 \mathrm{eV} /$ atom $)$ for metallic copper. It is interesting to note that $u_{\text {thes }}$ is in the similar range with the laser ablation threshold of $0.14 \mu \mathrm{J} / \mu \mathrm{m}^{3}[34]$ and the energy density for melting of $0.3 \mathrm{eV} /$ atom [35]. The melt limit is lower than $u_{\text {thes. }}$ This may relate to the similar dynamic effect used in the diffract-before-destroy scheme[36, 37], where a femtosecond x-ray pulse records diffraction pattern before the sample structure is lost. Similarly, melting does not proceed within the short pulse duration.

Approximating the attenuation length for $1-\mu \mathrm{J}$ pulse by the tabulated value of $4.5 \mu \mathrm{m}$ at $4.5 \mathrm{keV}$ [31], the absorbed energy density for the TPA experiment is estimated to be $u_{\exp }=0.093 \mu \mathrm{J} / \mu \mathrm{m}^{3}$. The estimation gives the upper bound of $u_{\text {exp }}$, because $\mu$ decreases as $u$ increases. Although this is higher than $u_{\text {thes }}$, the deviation of $\mu\left(u_{\text {exp }}\right)$ from $\mu\left(u_{\text {thres }}\right)$ is still small [Fig. 4(b)], and the TPA spectrum is considered to reflect the intrinsic electronic structure.

In summary, we successfully measured the x-ray TPA spectrum intrinsic to metallic copper. The spectrum can be explained qualitatively by the theory for metal with incomplete shells, indicating the higher sensitivity of TPA to the empty $3 \mathrm{~d}$ band. Since the 1s-3d transition is dipole-forbidden, x-ray TPA spectroscopy could easily reveal the complementary information to the conventional XAS. The $2 \mathrm{p}-3 \mathrm{~d}$ dipole transition is accessible in the soft $\mathrm{x}$-ray region [28], however, the hard $\mathrm{x}$ rays have advantages, such as longer probing depth and flexible sample environment. We also estimated the threshold to measure the intrinsic absorption spectrum, which is given by $u_{\text {thes }}$ for solids and not by $F_{\mathrm{c}}$. In the present study, the TPA spectrum was measured at slightly higher $u$ than $u_{\text {thes }}$. One could reduce $u$ using shorter pulses [38]. The Fourier-transform limit of the $0.42-\mathrm{eV}$ bandwidth is $4.3 \mathrm{fs}$, and using 4.3 -fs pulses, the 

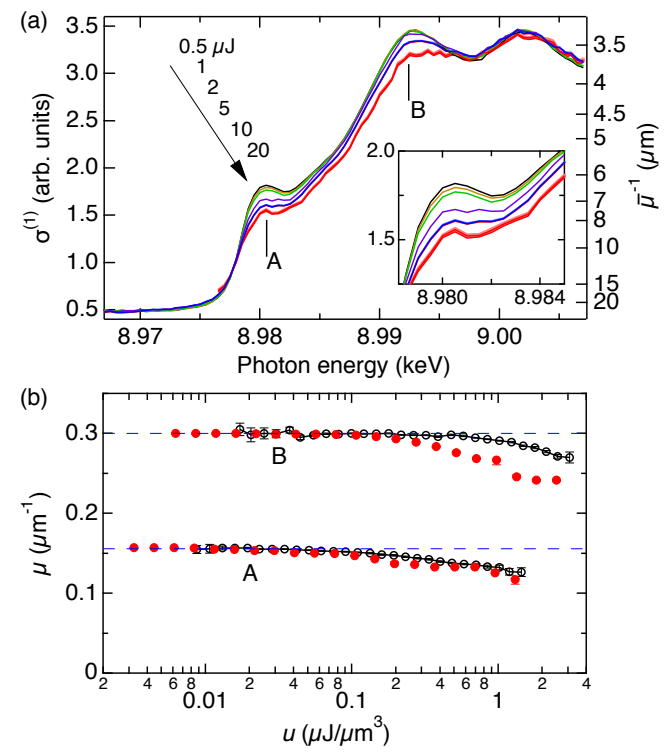

FIG. 4. (a) Pulse-energy dependence of OPA spectra. The OPA spectra are obtained from data measured at the pulse energies within $\pm 20 \%$ of the labeled value. The line width indicates the standard error of the mean. Inset shows magnified spectra around the peak A. (b) Estimated $\mu(u)$ (filled circles) at the peaks, A and B, in (a). Vertical bars represent the fitting error. Solid line with circles indicates $\bar{\mu}$ with the standard error of the mean. Dashed lines are guide for eyes.

same peak intensity can be realized at the half $u$ of the present study without degrading the photon-energy resolution. Furthermore, shorter pulses may relax $u_{\text {thes }}$ due to the dynamic effect in the secondary electron creation.

This work was supported by JSPS KAKENHI (15H03597). The experiment was performed with the approval of JASRI (Proposal No. 2015A8021, 2015B8001, 2016A8013, 2016B8039, 2017A8054).

* tamasaku@riken.jp

[1] G. Bunker, Introduction to XAFS (Cambridge University Press, Cambridge, 2010).

[2] P. Emma et al., Nature Photon. 4, 641 (2010).

[3] T. Ishikawa et al., Nature Photon. 6, 540 (2012).

[4] G. Doumy et al., Phys. Rev. Lett. 106, 083002 (2011).

[5] K. Tamasaku et al., Nature Photon. 8, 313 (2014).

[6] S. Ghimire et al., Phys. Rev. A 94, 043418 (2016).

[7] J. Szlachetko et al., Sci. Rep. 6, 33292 (2016).

[8] R. Boyd, Nonlinear Optics (Academic Press, San Diego, 2003).

[9] L. Young et al., Nature 466, 56 (2010).

[10] H. Fukuzawa et al., Phys. Rev. Lett. 110, 173005 (2013).

[11] K. Tamasaku et al., Phys. Rev. Lett. 111, 043001 (2013).

[12] A. Rudenko et al., Nature 546, 129 (2017).

[13] R. Santra and L. Young in Synchrotron Light Sources and Free-Electron Lasers, edited by J. R. Schneider and J. B. Hastings (Springer, Switzerland, 2014).

[14] H. Yoneda et al., Nat. Commun. 5, 5080 (2014).

[15] D. S. Rackstraw et al., Phys. Rev. Lett. 114, 015003 (2015).

[16] Y. Inubushi et al., Appl. Sci. 7, 584 (2017).

[17] See Supplemental Material at [URL will be inserted by publisher] for estimation of the power of each harmonic at the sample position.

[18] H. Yumoto et al., Nature Photon. 7, 43 (2013).

[19] K. Tono et al., New J. Phys. 15, 083035 (2013).

[20] T. Kameshima et al., Rev. Sci. Instrum. 85, 033110 (2014).

[21] F. Livet et al., Nucl. Instr. and Meth. A 451, 596 (2000).

[22] W. Zernik, Phys. Rev. 135, A51 (1964).

[23] F. T. Chan and C. L. Tang, Phys. Rev. 185, 42 (1969).

[24] S. A. Novikov and A. N. Hopersky, J. Pjys. B: At. Mol. Opt. Phys. 34, 4857 (2001). 
[25] A. Sytcheva, S. Pabst, S.-K. Son and R. Santra, Phys. Rev. A 85, 023414 (2012).

[26] A. L. Ankudinov, B. Ravel, J. J. Rehr and S. D. Conradson, Phys. Rev. B 58, 7565 (1998).

[27] G. Kresse and J. Furthmüller, Phys. Rev. B 54, 11169 (1996).

[28] F. de Groot and A. Kotani, Core Level Spectroscopy of Solids (CRC Press, New York, 2008).

[29] A. Kotani and Y. Toyozawa, J. Phys. Soc. Jpn. 35, 1073 (1973).

[30] P. W. Anderson, Phys. Rev. Lett. 18, 1049 (1967).

[31] B. L. Henke, E. M. Gullikson and J. C. Davis, At. Data Nucl. Data Tables 54, 181 (1993).

[32] S. P. Hau-Riege, High-Intensity X-rays-Interaction with Matter (Wiley-VCH, Weinheim, 2011).

[33] B. Ziaja, R. A. London and J. Hajdu, J. Appl. Phys. 97, 064905 (2005).

[34] M. Hashida et al., Proc. SPIE 4423, 178 (2001).

[35] J. Arthur et al. Linac Coherent Light Source (LCLS) Conceptual Design Report SLAC-R-593 (Stanford, 2002 ).

[36] R. Neutze, R. Wouts, D. van der Spoel, E. Wechert and J. Hajdu, Nature 406, 752 (2000).

[37] H. N. Chapman, C. Caleman, N. Timneanu, Phil. Trans. R. Soc. B 369, 20130313 (2014).

[38] S. Huang et al, Phys. Rev. Lett. 119, 154801 (2017). 